\title{
Educación para la innovación agraria. Un modelo para la acción educativa' ${ }^{1}$
}

Pau Pérez Ledo²

\section{RESUMEN}

Este estudio se centra en la figura del productor agrario valenciano y su comportamiento frente a la innovación. Adoptar una postura proclive a la innovación depende de múltiples factores que influyen en la gestión de la empresa agraria. Este estudio utiliza los datos procedentes de una encuesta a productores agrarios valencianos, diseñada ad hoc para la investigación, que profundiza en los factores socioeconómicos que pueden decantar al productor agrario hacia una postura favorable a la innovación. Posteriormente, el estudio se centra en obtener un perfil tipo que caracteriza al productor según sus características y las de su explotación, según su actitud innovadora y la orientación hacia factores influyentes, como son la orientación al mercado y la orientación al aprendizaje. Se observa una asociación positiva entre la actitud innovadora y el nivel de formación del agricultor y su proximidad a zonas urbanas, donde existe más acceso a conocimiento y servicios.

\section{PALABRAS CLAVE:}

educación, innovación, productor agrario, agricultura valenciana, actitud innovadora, orientación al mercado, orientación al aprendizaje, encuesta.

$1 \quad$ Agradecimientos: Los autores agradecen el apoyo del Plan Nacional de I+D a este artículo en el marco del proyecto AGL2012-397

$2 \quad$ Universitat de València. Departament Sociologia i Antropologia Social. 


\section{ABSTRACT}

Farmers are the main actors of innovation in the agricultural sector. This study focuses on the socio-economic profile of the Valencian farmer and his/her attitude towards innovation. Adopting a position inclined to innovate depends on multiple factors that influence bussiness management. This paper uses data from a farmers' survey carried out in the Valencia region, which was designed ad hoc for the research, that considers the socioeconomic factors that make farmers take a position favorable to innovation. Later, the study focuses on a typical profile of innovative producers, according to their characteristics, taking into account market orientation, learning orientation and innovative attitude. The results show a positive contribution of level of education and location in urban areas [access to knowledge and services] to the innovative attitude.

\section{KEYVORDS:}

education, valencian agriculture, innovative attitude, market orientation, learning orientation, survey. 


\section{INTRODUCCIÓN}

Como el resto de sectores, el sector agroalimentario ha tenido que enfrentarse a sus propios retos, inmerso en un escenario que cambia con rapidez. Se considera un sector con influencia en el desarrollo del territorio, por sus funciones de suministrador de alimentos y su alta ocupación del mismo, como muestra una reciente tesis defendida en el Departamento de Economía y Ciencias Sociales de la Universitat Politècnica de València (López-García et al, 2012). Tradicionalmente, la literatura identifica al sector como de baja intensidad innovadora (Connor y Chiek, 1997). Estudios recientes refutarían los resultados anteriores, mostrando que el conjunto del sector agroalimentario valenciano posee una intensidad innovadora mayor que otros sectores del mismo territorio (Alba, 2012), aunque ésta sea menor que en el conjunto nacional. Sin embargo, al diferenciar los resultados según se trate del sector primario o industria agroalimentaria, se pone de relieve que el primero acaba resultando de baja intensidad innovadora en relación a la industria en la Comunidad Valenciana.

Teniendo el sector agrario valenciano en general una baja intensidad innovadora, los vínculos intersectoriales provocan una innovación indirecta -o incorporada en los productos/servicios que el sector adquiere-, proveniente de otros sectores económicos. Estudios recientes [Alba et al, 2010) indican que la innovación indirecta es importante en el sector primario, mucho más que en la industria agroalimentaria, y que, además, esta importación de conocimiento se realiza predominantemente desde otros territorios del país.

Cabe preguntarse el motivo de esta baja intensidad innovadora en el sector agrario, ya que no deja de ser curiosa la diferencia respecto a la industria alimentaria (ambos ubicados en la Comunidad Valenciana]. ¿Se debe a factores internos, derivados de la propia estructura del sector, que rigidiza o dificulta la incorporación de innovaciones? ¿Es debidoa una percepción derrotista por parte del productor agrario, responsable de la iniciativa innovadora en su explotación? Si esto fuera cierto, ¿qué influye en la percepción del productor? ¿Factores sociológicos, individuales...? ¿O es quizá la compleja estructura del mercado la que limita el margen de maniobra de un productor que realmente está motivado? La presente investigación atiende a los factores que la literatura identifica como claves en el proceso de toma de decisiones relativas a innovación con el fin de elaborar el perfil "tipo" del productor valenciano innovador. El objetivo principal de la investigación es identificar las características que definirían el perfil del productor agrario valenciano innovador, analizando información obtenida de primera mano mediante una encuesta al productor diseñada ad hoc para la investigación.

\section{RESTRICCIONES ESTRUCTURALES}

De entre todos los rasgos que diferencian la agricultura valenciana de sus homónimas el minifundismo ha sido uno de los temas de debate más presentes en la literatura. Es tan inherente al campo valenciano, que un $40 \%$ de las explotaciones poseen menos de una hectárea. Bajo estas circunstancias la tierra se ha convertido en un factor de producción escaso en los planteamientos empresariales. Las explotaciones no han podido evolucionar hacia un tamaño mínimo que permita aprovechar la economía de escala. En el año 2009 cerca del 54\% de las explotaciones poseían menos de dos hectáreas. La evolución entre los Censos de 1999 y 2009 muestra que desciende el número de explotaciones con menos de una hectárea y aumenta el número de explotaciones de 
los intervalos superiores. La explicación puede estar en el descenso del número de explotaciones, lo que indicaría un abandono de la agricultura por parte de otros agricultores; estas tierras, que se quedan sin explotar, son adquiridas por el resto de explotaciones, que aumentan su tamaño.

La literatura coincide en enmarcar otra particularidad del sector valenciano, que es causa y consecuencia de la agricultura a tiempo parcial. Arnalte [1989] denominaba a este proceso la "desactivación de explotaciones", refiriéndose al cambio paulatino hacia la externalización de gran parte de las tareas agrícolas adentrándose en un modelo de organización productiva, típica de regadío, que permite el manejo de una mayor superficie por cada persona ocupada. Este modelo va aparejado con la agricultura a tiempo parcial y permite mantener la estructura minifundista de las explotaciones, en lugar de promover la concentración para alcanzar mayores tamaños.

En la actualidad vemos que los problemas que describieron los economistas y geográfos valencianos para la agricultura valenciana en los años ochenta y noventa del siglo XX persisten. Sin embargo, según pasan los años se puede apreciar una evolución tímida en los indicadores que muestran el esfuerzo del sector en pro de alcanzar un equilibrio. Por ejemplo, la estructura minifundista se ha visto modificada por la dinámica del sector, que ha permitido que las explotaciones del interior tiendan a reestructurarse. La pérdida de población ocupada que se ha experimentado con los años ha permitido cierta concentración de las explotaciones, por lo que se puede observar un aumento del tamaño medio. En términos generales, la Comunidad Valenciana ha perdido en 10 años casi el 47\% de explotaciones, según el Censo Agrario [1999-2009, INE). No obstante, la agricultura litoral ha mantenido su alto grado de minifundismo.

\section{FACTORES DE INNOVACIÓN}

El estudio de la innovación no es nuevo en el análisis de las ciencias sociales aplicadas a la agricultura y el mundo rural. Sin embargo, pocos estudios integran el análisis del comportamiento de un sujeto principal de la innovación: el agricultor.

La metodología empleada en el presente trabajo se basa en los datos obtenidos mediante una encuesta que fue realizada durante el segundo semestre del año 2011. Se pretende recopilar información acerca de factores de interés como: las características sociodemográficas del productor [factores individuales], de su explotación [factores organizativos] y de todos los constructos $^{3}$ que puedan constituir una definición del comportamiento innovador o influir en la toma de decisiones relativas a la innovación.

Posteriormente, el estudio se centrará en examinar las relaciones entre tres factores o constructos $^{4}$ que resumen el comportamiento innovador del productor agrario valenciano, que son:

- la orientación al mercado

- la orientación al aprendizaje

- la actitud innovadora,

3 El constructo representa un concepto abstracto cuya definición es difícil de establecer y que precisa de medidas indirectas para su estudio (Pérez-Gil et al. 2000]. 
Estos factores, reflejados en la Figura 1, constituyen en sí mismos una descripción de las dimensiones que componen la conducta innovadora. Las innovaciones son el vehículo mediante el cual la ciencia se transforma para incorporarse a las empresas y crear valor. El empresario tiene que mostrar una orientación favorable al aprendizaje para modificar su actividad y acometer innovaciones de todo tipo (producto, proceso, organización, función, etc). Adicionalmente, los estudios resaltan la importancia de adoptar una estrategia empresarial basada en la orientación al cliente que, combinándola con una actitud innovadora, mejora la competitividad de las empresas y su integración en el entorno económico. Podemos aceptar el hecho de que existe una retroalimentación entre actitud innovadora y orientación al mercado, de manera que la comunicación empresa-cliente fomenta la puesta en marcha por parte de la empresa de los productos/servicios que el cliente demanda. Dicho de otra manera, incorporar en la planificación empresarial el conocimiento de las necesidades de los clientes, incide en innovaciones más acertadas, con mayor probabilidad de éxito en el mercado, y por tanto menor riesgo para el emprendedor que se encarga de introducirlas. De inmediato toman protagonismo factores como el conocimiento o el mercado. El eslabón que une ambos es el innovador o emprendedor que ha de tener sendos vínculos con el conocimiento y con su cliente, según el esquema de la Figura 1.

Figura 1: Aplicación de innovaciones por parte del responsable empresarial

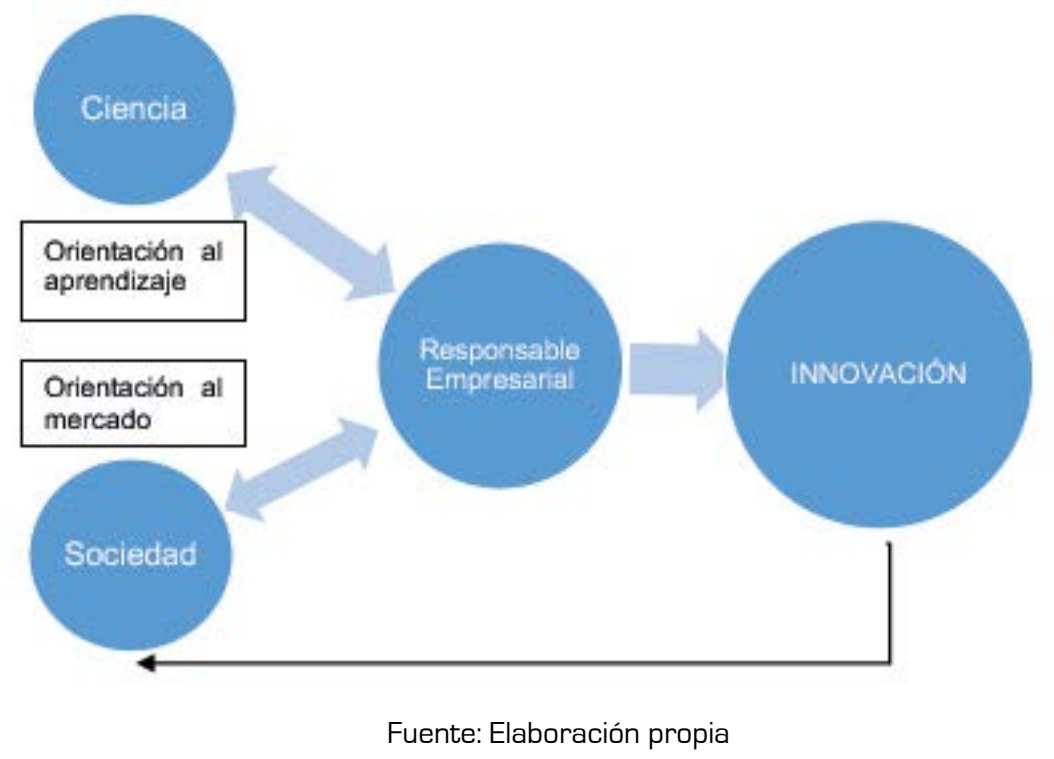

Existe una carencia de estudios preliminares que deja múltiples interrogantes a nivel de productor; ¿qué percepción tiene de la innovación? ¿cómo influyen los factores como la orientación al mercado en la actitud innovadora?; o por el contrario ¿influye la orientación al mercado en la actitud innovadora? En definitiva, ¿qué determina el comportamiento innovador en el productor agrario valenciano?

Y respecto a los factores más relacionados con las características del individuo y con las de su explotación, ¿es de suponer que también ejercen influencia sobre la actitud innovadora? Hay ciertos factores que favorecen una actitud innovadora sea cual sea el sector al que se aplique, ¿podrían extrapolarse al sector agrario valenciano? ¿cómo influiría, por ejemplo, la edad o el sexo en la actitud innovadora? 
Los constructos no son variables objetivas que se midan directamente, como podría ser la edad del empresario, sino que se trata de aspectos relacionados con actitudes personales que conforman un concepto complejo, abstracto y subjetivo. En realidad, un constructo se compone a su vez de múltiples facetas virtualmente imposibles de medir con una sola variable o indicador así que, ¿cómo efectuar la medición de un concepto tan vaporoso y complejo?

La técnica más empleada para medir estos constructos es la escala Likert, llamada así por Renis Likert, que la propuso por primera vez en la década de 1930 (Corbetta, 2003, p.233). En realidad se trata de la técnica más utilizada para medir aspectos de la conducta, como puede ser la satisfacción de un cliente, ya que el procedimiento de Likert es muy intuitivo. Consiste en asignar a cada constructo una serie de afirmaciones o ítems que el encuestado manifestará su grado de acuerdo o en desacuerdo.

Nuestra encuesta a agricultores valencianos diseñó una serie de afirmaciones sobre cada uno de los constructos. El encuestado reflejó su grado de acuerdo puntuando cada una de esas afirmaciones en una escala de 1 a 7, siendo 1 "completamente en desacuerdo" y 7 "completamente de acuerdo". En cada una de las afirmaciones se procuró reflejar un aspecto o dimensión del constructo que estamos estudiando. Por ejemplo, el constructo "Actitud Innovadora" puede medirse según el nivel de acuerdo que refleje el encuestado respecto a esta afirmación:

\section{"Adoptar innovaciones es una decisión útil"}

Afirmación que puede traducirse, con bastante seguridad, como el grado de asociación que establece el encuestado entre innovar y utilidad. Así pues estamos midiendo la actitud innovadora mediante una de sus facetas: la utilidad. Pero previsiblemente la actitud innovadora no posee exclusivamente ese significado así que, en base al razonamiento teórico, decidimos incluir otras afirmaciones para reforzar la medición del constructo. Así, por ejemplo, en la Tabla 1 mostramos una serie de afirmaciones a título de ejemplo que vendrían a reflejar el constructo "orientación al mercado".

Tabla 1. Ejemplo de variables Likert para medir la "Orientación al mercado"

\begin{tabular}{|c|c|c|c|c|c|c|c|c|}
\hline \multirow[b]{2}{*}{ Sigo las orientaciones de calidad que transmite el cliente. } & \multicolumn{4}{|c|}{ En desacuerdo } & \multicolumn{3}{|c|}{ De acuerdo } & \\
\hline & 1 & 2 & 3 & 4 & 5 & 6 & 7 & NS \\
\hline Cada año busco nuevos clientes & 1 & 2 & 3 & 4 & 5 & 6 & 7 & NS \\
\hline Los clientes me orientan sobre las variedades a plantar & 1 & 2 & 3 & 4 & 5 & 6 & 7 & NS \\
\hline $\begin{array}{l}\text { Mi preocupación por la calidad me da una ventaja sobre otras } \\
\text { explotaciones. }\end{array}$ & 1 & 2 & 3 & 4 & 5 & 6 & 7 & NS \\
\hline $\begin{array}{l}\text { Mi preocupación por ofrecer productos más baratos me da una } \\
\text { ventaja sobre otras explotaciones }\end{array}$ & 1 & 2 & 3 & 4 & 5 & 6 & 7 & NS \\
\hline
\end{tabular}

Fuente: Elaboración propia 
Adicionalmente, nuestra encuesta incorporó preguntas sobre datos más objetivos que han sido de utilidad tanto para segmentar a la población como para explicar el comportamiento innovador. Se trata de variables socioeconómicas con un sentido más definido, que reflejan factores individuales y organizativos que pueden influir en el comportamiento innovador [Tabla 2).

Tabla 2. Listado de características individuales y organizativas

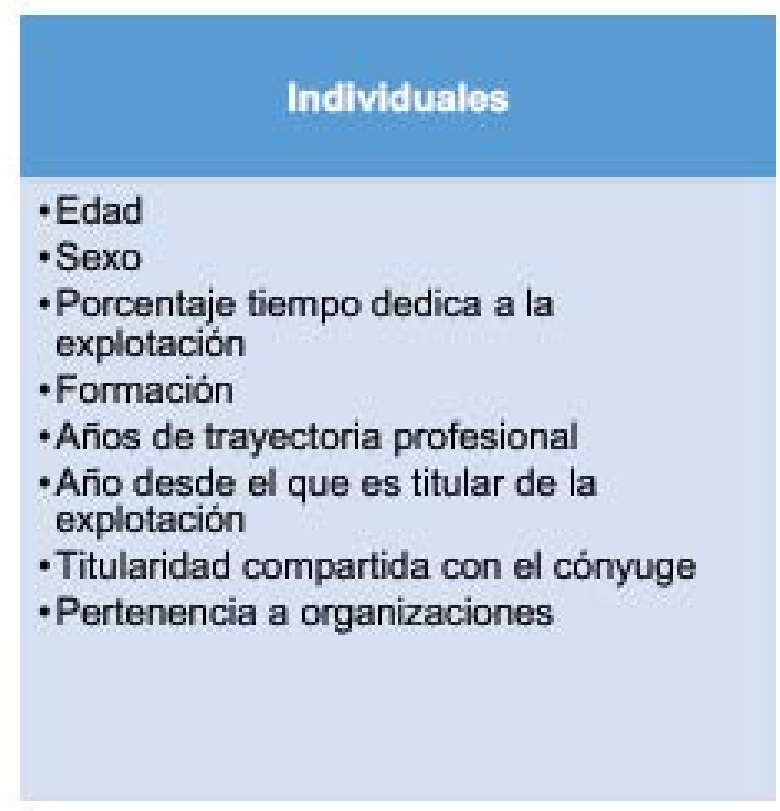

\section{Organizativos}

- Tamaño de la explotación (Hectáreas cultivo/cabezas)

-Año desde el que existe la explotación

- Ayuda familiar

- Contrata asalariados

- Régimen de tenencia

- Sisterna de riego

- Agricultura ecológica

- Destino de la producción

- Cliente preferente y acuerdos contractuales

- Margen Bruto de la explotación

-Actividades complementarias

Fuente: Elaboración propia

\section{CARACTERÍSTICAS SOCIOECONÓMICAS}

Tras unos meses de recogida de información se creó una base de datos codificada, con 253 encuestas válidas que permitieron iniciar los primeros análisis, meramente descriptivos, destinados a conocer la muestra de estudio y observar las diferencias respecto a la población con datos oficiales; en definitiva, enmarcando la muestra dentro de su población, conocida en parte gracias a las estadísticas disponibles.

Así pues, se constata que los datos de estudio corresponden a una muestra en general un poco más joven de lo que plasman las estadísticas agrarias oficiales; la media de edad se sitúa en torno a los 50 años, lo que sigue reflejando una población relativamente envejecida. La masculinización del mundo rural se deja sentir en los resultados, que son algo más extremos de lo que cabía esperar. Ciertamente el número de mujeres encuestadas es bajo, un 9\% del total, lo cual se debe posiblemente al haber tomado como base una muestra de afiliados a organizaciones agrarias y cooperativas.

En lo que respecta al perfil del encuestado, se trata de un productor en general implicado en su explotación, que dedica más del 50\% de su tiempo a la explotación (un 74\% de ellos lo hace). La formación con la que cuenta oscila entre el graduado escolar y formación a nivel de bachillerato 
o FP, aunque también hay un 13\% de titulados universitarios. En cuanto a su experiencia como titular de explotaciones agrarias, ésta es amplia, de media 22 años, con la particularidad de que también son muchos los años que lleva gestionando la explotación vinculada al cuestionario. Eso sí, compaginándolo a lo largo de su vida con el trabajo en otros sectores distintos al agrario.

Las cooperativas o SATs aglutinan a la mayoría de encuestados que declararon pertenecer a alguna asociación agraria, un 67\%, así como las comunidades de regantes, un $45 \%$, y las organizaciones profesionales agrarias, un 29\%.

En la Comunidad Valenciana la producción agrícola vegetal supone la mayor parte de la producción agraria final. La muestra sigue un patrón similar presentando un $80 \%$ de explotaciones que son agrícolas, un 15\% que también tienen ganadería y finalmente un 3'5\% de explotaciones mayoritariamente ganaderas. También hay representación próxima a la realidad agraria en los cultivos, con los cítricos en el primer puesto por superficie, horticultura, viñedo, olivar y almendro entre otros caracterizando la diversidad agraria de la Comunidad. Por su parte el ganado porcino es la cabaña ganadera más presente en la muestra, seguida del sector avícola.

En cuanto a la mano de obra que trabaja en la explotación aproximadamente la mitad de los encuestados declara contratar asalariados. Otra mitad, aproximadamente, también declara hacer uso de familiares para las tareas agrarias. El riego por goteo está muy extendido entre la muestra ya que el 60\% lo tiene implantado en su terreno. Otro factor que define a la explotación es el destino de la producción; aquí la cooperativa vuelve a destacar, obviamente, pero también se constata el destino a comercios mayoristas. Esta no es una característica excluyente y los encuestados hacen uso de varias vías simultáneas para dar salida a sus productos. El margen bruto que engloba a casi el $60 \%$ de las explotaciones se encuentra en el rango comprendido entre 20.000 y $50.000 € /$ año.

\section{4. ¿QUÉ PIENSAN LOS AGRICULTORES?}

Las preguntas Likert se han agrupado en dos grandes categorías: las que tienen que ver con la orientación al mercado y al aprendizaje de los agricultores, por un lado, y las que reflejan una actitud positiva hacia el hecho de innovar, por el otro. En cierta medida, siguiendo el esquema de la Figura 1, la primera categoría representa la propensión del agricultor para captar información del exterior y ser capaz de asimilarla, mientras que la segunda ya denota el carácter más o menos innovador del agricultor. Posteriormente, mediante la técnica de componentes principales, se ha subdividido a su vez la primera categoría de variables, en dos constructos: orientación al mercado y orientación al aprendizaje.

La siguiente tabla muestra los estadísticos descriptivos de cada una de las variables Likert que componen los constructos. 
Tabla 3. Estadísticos descriptivos de las variables Likert

\begin{tabular}{|c|c|c|c|c|}
\hline & VARIABLE & $\mathrm{N}$ & Media & $\begin{array}{l}\text { Desv. } \\
\text { tip. }\end{array}$ \\
\hline \multirow{12}{*}{ 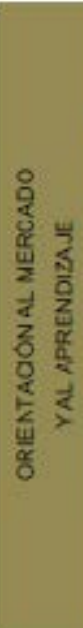 } & Siqo las orientaciones de calidad que transmite el cliente & 214 & 5,77 & 1,50 \\
\hline & Cada año busco nuevos clientes & 191 & 3,42 & 2,26 \\
\hline & Los clientes me orientan sobre las variedades a plantar & 197 & 3,43 & 2,23 \\
\hline & Mi preocupación por la calidad me da una ventaja sobre ofras explotaciones & 221 & 5,22 & 1,87 \\
\hline & $\begin{array}{l}\text { Mi preocupación por ofrecer productos más baratos me da una ventaja sobre } \\
\text { otras explotaciones }\end{array}$ & 199 & 3,03 & 1,97 \\
\hline & La satisfacción del cliente es el principal objetivo de mi empresa & 218 & 5,72 & 1,68 \\
\hline & Me gusta leer revistas sobre nuevos cultivos o métodos que podria introducir & 235 & 5,56 & 1,64 \\
\hline & Me gusta asistir a ferias, cursos o jornadas para conocer nuevas ideas & 238 & 5,65 & 1,56 \\
\hline & $\begin{array}{l}\text { Mis empleados y miembros de mi familia consideran que el aprendizaje es } \\
\text { importante }\end{array}$ & 222 & 5,63 & 1,55 \\
\hline & Intercambio experiencias con otros agricultores & 239 & 5,95 & 1,23 \\
\hline & Cuando una nueva técnica/producto no da resultados analizo las causas del fallo & 233 & 5,67 & 1.49 \\
\hline & Me siento corresponsable de los fallos en mi explotación & 241 & 6,05 & 1,25 \\
\hline \multirow{6}{*}{ 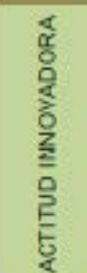 } & Adoptar innovaciones es una decisión útil & 241 & 5,16 & 1,84 \\
\hline & Valoro a las personas que innovan & 248 & 5,97 & 1,30 \\
\hline & Las personas que son importantes para mi piensan que yo debo innovar & 226 & 4,90 & 1,78 \\
\hline & Estoy motivado para innovar & $24 \pi$ & 4,57 & 2,15 \\
\hline & Las Innovaclones mejoran los resultados de mi explotación & 242 & 5,12 & 1,86 \\
\hline & Innowar merece la perla & 241 & 5,16 & 1,84 \\
\hline
\end{tabular}

Fuente: Elaboración propia

Respecto a las puntuaciones, la media más alta es un 6'05 que corresponde a la variable "Me siento corresponsable de los fallos en mi explotación".

Las puntuaciones más altas reflejan una actitud innovadora que se muestra como aquella que sabe apreciar el esfuerzo que conlleva innovar ["valoro a las personas que innovan", 5'97], mientras que el mercado ocupa un lugar destacado en los planteamientos empresariales ["sigo las orientaciones de calidad que transmite el cliente", 5'77, y "la satisfacción del cliente es el principal objetivo de mi empresa", 5'72). Así se confirma la orientación al mercado que lleva caracterizando a la agricultura valenciana durante décadas y cómo este mercado es el principal demandante de innovaciones, aspecto que es claramente percibido por el agricultor.

Por otra parte, el productor muestra un alto grado de implicación en la explotación ["cuando una nueva técnica/producto no da resultados analizo las causas del fallo", 5’67].

Otro aspecto positivo es la predisposición al aprendizaje mediante la cooperación, aunque a un nivel superficial, con otros productores ["Intercambio experiencias con otros agricultores", 5'95]. Resulta ilustrativo que el tándem cooperación/competitividad se incline hacia el lado de la cooperación. Esto indica que se percibe como beneficiosa la cooperación. Lo que quiere decir que pesan más las ventajas obtenidas, dejando en un segundo lugar al recelo de la competitividad empresarial. Por otra parte, también es un indicador de la voluntad de aprender. Y aprender se lleva a cabo de la manera más accesible, con productores que trabajan con el mismo producto ya 
que es habitual a lo largo de la geografía valenciana que existan zonas dedicadas prácticamente al monocultivo de una especie. Aunque bien puede significar que el agricultor echa mano de la información más accesible ante la carencia de otras vías para la transferencia de conocimiento, como veremos a continuación.

En lo que respecta a los aspectos con puntuaciones más bajas, el productor percibe algunos factores externos como factores que dificultan un entorno propicio para la innovación. El precio de mercado es una variable que el productor no puede controlar ["mi preocupación por ofrecer productos más baratos me da una ventaja sobre otras explotaciones", 3'03] y la respuesta refleja una preocupación pesimista acerca de la competencia vía precios.

Como conclusión se puede destacar la implicación del productor con su actividad y cómo es consciente de la importancia de sus clientes. Además existe predisposición por parte del productor a implicarse en el proceso innovador. Otras preguntas de la encuesta reflejaron que el entorno socioeconómico del agricultor no es percibido como favorable para tales actuaciones. Bien sea por desconocimiento o por carencia lo cierto es que el productor no percibe que tenga mecanismos públicos o privados en los que pueda apoyarse fácilmente y ve más accesible la información que le proporcionan otros productores.

\section{MEDIR LOS CONSTRUCTOS}

El siguiente paso de la investigación consistió en simplificar la información, perdiendo la menor cantidad de información posible. La técnica seleccionada para este propósito fue el análisis de componentes principales (ACP). La técnica estudia la estructura de las interrelaciones dadas entre un gran número de variables, con el fin de hallar dimensiones subyacentes, conocidas como componentes [en realidad, los indicadores sintéticos de los tres constructos definidos por una serie de preguntas de la encuesta]. La técnica da como resultado una serie de nuevas variables, que serán la representación del componente hallado en la estructura de las variables.

El ACP es un procedimiento que tiene como objetivo el análisis de la variación total que una variable comparte con otras variables, para poder resumir la información contenida en el conjunto total de variables interrelacionadas. La ventaja que otorga el ACP, es que reduce la información disponible creando nuevas variables que son combinaciones lineales del conjunto inicial perdiendo la menor cantidad posible de la variabilidad inicial. La técnica permitió definir en forma de componentes o variables sintéticas, los tres constructos: Orientación al Mercado, Orientación al Aprendizaje, y Actitud Innovadora.

En nuestro caso, diez variables Likert de la encuesta fueron sintetizadas en dos componentes correspondientes a los constructos Orientación al Mercado y Orientación al Aprendizaje, que en conjunto explicaban un $57 \%$ de la varianza original de las variables originales. Se definió el componente correspondiente al constructo Actitud Innovadora a partir de seis variables Likert de modo que dicho componente principal explicaba el 60\% de la varianza inicial de las variables iniciales. 
Figura 2. Obtención de variables representativas de los constructos

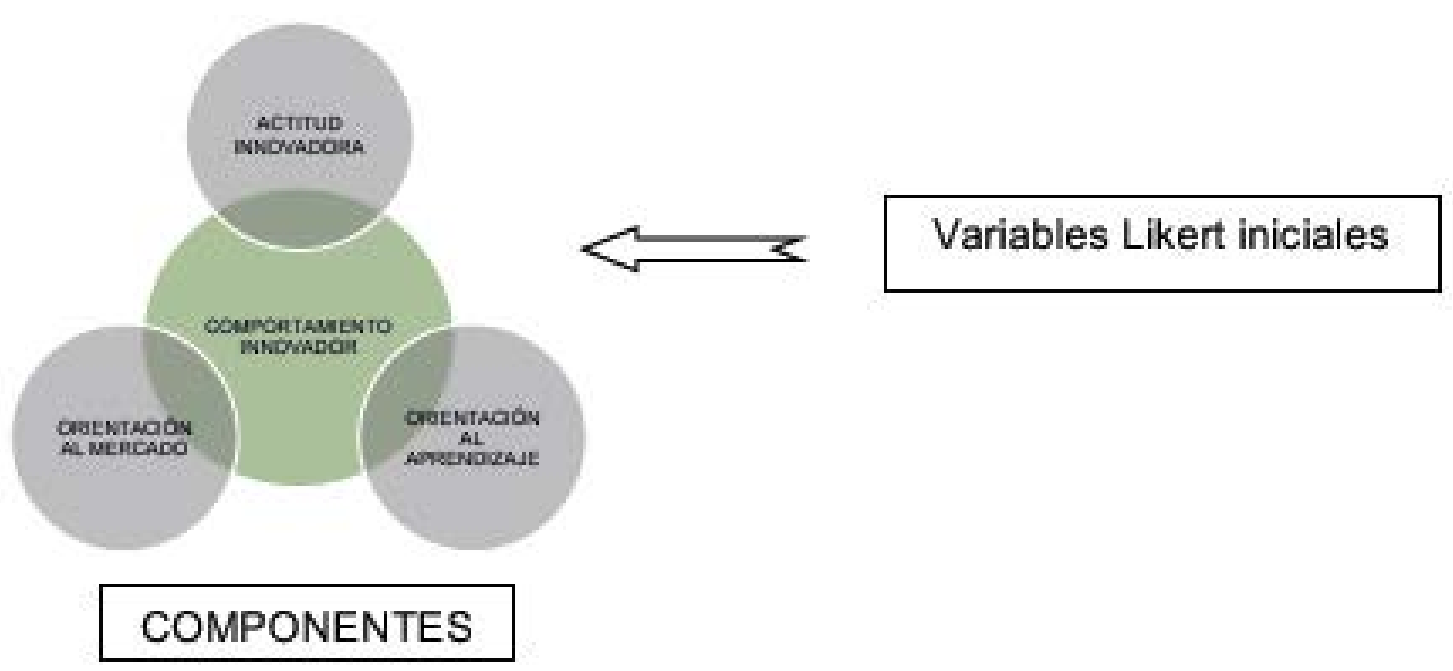

Fuente: Elaboración propia

\section{PERFILES INNOVADORES DE LOS AGRICULTORES VALENCIANOS}

Una vez obtenidas las variables representativas de los constructos de estudio se aplicaron procedimientos estadísticos destinados a conocer si existía diferencia entre las puntuaciones medias de diferentes grupos respecto a los mismos. El uso de las técnicas multivariantes permitió realizar un análisis conjunto de varias variables con el fin de encontrar un modelo que explique el comportamiento innovador, por lo que se aplicaron modelos de regresiones múltiples incorporando en cada uno de ellos, como variables exógenas, las características individuales y organizativas que figuran en la Tabla 2.

A partir de estos resultados se ha podido catalogar al productor agrario innovador según las características socioeconómicas que favorecen su inclinación hacia cada uno de los tres constructos estudiados. Se han agrupado [ver Tabla 4] los resultados según el nivel de estudios del encuestado y su localización en medio rural, urbano o intermedio siguiendo los criterios de ruralidad de la OCDE y la clasificación de sistemas territoriales de Boix y Galetto [2008] modificado por López Estornell [2010].

En síntesis, la siguiente figura ilustra los perfiles que se pueden establecer. 
Figura 3. Perfiles de agricultores según su posición en los tres factores de innovación identificador: Orientación al Mercado, Orientación al Aprendizaje y Actitud Innovadora

\section{Débilmente Innovadores}

\section{Actitud Innovadora, Orientación al Mercado y Orientación al Aprendizaje $(\downarrow)$}

\section{Innovadores}

\section{Actitud Innovadora, Orientación al Mercado y Orientación al Aprendizaje ( $)$}

\section{Innovadores Intermedios}

3.1

3.2

Actitud Innovadora $(\downarrow)$ y alguna orientación $(\uparrow) \quad$ Actitud Innovadora $(\uparrow)$ y alguna orientación $(\downarrow)$

Fuente: Elaboración propia

\section{a) Débilmente innovadores}

Aquellos casos que muestran una orientación desfavorable hacia los tres componentes del comportamiento innovador. En nuestra muestra constituyen el 11,7\% de los entrevistados [Tabla 4). Hay que tener en cuenta que los agricultores encuestados son personas que colaboran con organizaciones profesionales por lo que es lógico que el colectivo débilmente innovador no tenga una presencia mayoritaria en la muestra. En la tabla sí se comprueba que en este grupo son mayoritarias las explotaciones donde el entrevistado no dispone de estudios o sólo de estudios básicos. Además, son explotaciones que se localizan en un medio predominantemente rural, lo que pone en evidencia los handicaps existentes en una parte del territorio y la pertinencia de las políticas de desarrollo rural. En este grupo se encuentran muchas explotaciones cuya producción es predominantemente ganadera. Los resultados empresariales también influyen en el comportamiento innovador, de manera que aquellas que no superan los $5.000 €$ anuales de margen bruto aproximado tienen una probabilidad mayor de estar presentes en esta categoría. También el modelo de agricultor a tiempo parcial está presente en este perfil.

\section{b] Innovadores}

Aquellos casos que muestran una orientación favorable hacia los tres componentes del comportamiento innovador. Se observa que en este grupo, cuya participación en el total de encuestados es del 28,3\%, existen características opuestas al grupo anterior, con presencia mayoritaria [aunque no exclusiva] de agricultores con estudios más allá de los básicos y localización en zonas urbanas. Este grupo cuenta con una mayor presencia relativa de explotaciones cuya 
producción se dedica a cultivos hortícolas o cítricos. Contar con infraestructura para riego por goteo también posiciona a las explotaciones en el grupo de innovadores. En cuanto a los resultados empresariales, en contraposición a los pertenecientes al grupo de débilmente innovadores, presentar un margen bruto anual superior a 50.0000 es una característica que influye positivamente para posicionarse en el grupo de explotaciones innovadoras.

En cuanto a las características sociodemográficas del productor, el productor "tipo" de esta categoría dedica más de la mitad de su tiempo a la explotación y ha recibido formación. Las explotaciones que tienen toda o parte de su suelo en régimen de arrendamiento muestran una orientación positiva hacia esta categoría. Es destacable que pertenecer a cooperativas también influye positivamente en el comportamiento innovador, de manera que los productores que pertenecen a alguna asociación en la muestra tienden a mostrar no sólo una mejor actitud innovadora, sino mejores orientaciones al aprendizaje y al mercado.

\section{c] Innovadores intermedios}

Son dos categorías en los que las explotaciones presentan un posicionamiento favorable en algunos de los constructos sin llegar a ser favorable para todos ellos. Es llamativo que una de estas categorías "Innovador intermedio (b]" es la mayoritaria [un 32,4\% de los encuestados], con agricultores con una actitud innovadora apreciable pero relativamente baja orientación al mercado o al aprendizaje. En este caso, el problema no es la propensión a asumir cambios, sino a percibir las señales del mercado, incluso cuando el nivel de formación mayoritario [un 63\%] es superior al básico y su localización es mayoritariamente urbana. Aquí se detecta la carencia de unos prerrequisitos que la política agraria y los sistemas de formación deberían de contrarrestar. En el "Innovador intermedio [a]" se confirma que un nivel menor de formación y la lejanía a zonas urbanas no están asociadas a una mayor actitud innovadora. En este grupo tienden a tener una mayor presencia relativa explotaciones orientadas a cultivos leñosos, cuya fijeza puede explicar en parte la baja propensión a innovar. Se observa que en este grupo la edad del productor tiende a ser mayor, lo que influye negativamente sobre todo en la orientación al aprendizaje y en la actitud innovadora.

Tabla 4. Perfiles innovadores, nivel de formación y localización de las explotaciones

\begin{tabular}{rrrrrr} 
& $\begin{array}{c}\text { Débilmente } \\
\text { innovadores }\end{array}$ & $\begin{array}{r}\text { Innovador } \\
\text { Innovadores }\end{array}$ & $\begin{array}{c}\text { Innovador } \\
\text { intermedio } \\
\text { (a) }\end{array}$ & $\begin{array}{c}\text { intermedio } \\
\text { (b) }\end{array}$ \\
\hline Formación & $\%$ & $11,7 \%$ & $28,3 \%$ & $27,6 \%$ & $32,4 \%$ \\
\hline $\begin{array}{r}\text { Sin estudios reglados, } \\
\text { Graduado escolar } \\
\text { Bachillerato, FP, } \\
\text { Universitario }\end{array}$ & $58,8 \%$ & $41,2 \%$ & $53,7 \%$ & $50,0 \%$ & $37,0 \%$ \\
\hline Ristema Territorial & Rural & $75,0 \%$ & $30,0 \%$ & $53,8 \%$ & $34,1 \%$ \\
\hline Urbano & $25,0 \%$ & $62,5 \%$ & $38,5 \%$ & $59,1 \%$ \\
\hline
\end{tabular}

Innovador intermedio (a) Actitud innovadora $\downarrow$ y alguna orientación $\uparrow$

Innovador intermedio (b) Actitud innovadora $\uparrow$ y alguna orientación $\downarrow$

Fuente: Elaboración propia 


\section{RESUMEN Y CONCLUSIONES}

En términos generales la presente investigación ha dotado al conocimiento de un nuevo punto de referencia para avanzar en el estudio de la innovación en el sector agrario valenciano. El estudio de los productores implicados en la actividad que desarrollan muestra el perfil de los que realmente adoptan una actitud fructífera para conseguir que el sector se integre plenamente en la dinámica económica actual.

Así, hemos podido observar que las carencias estructurales que fueron objeto de estudio a finales del siglo $X X$ continúan presentes en la agricultura valenciana, si bien es cierto que se puede observar una tímida evolución con el propósito de paliarlas.

Los resultados de la investigación muestran las características socioeconómicas del productor y su explotación que favorecen el comportamiento innovador, entendido éste como la combinación de tres componentes o constructos, orientación al mercado, orientación al aprendizaje y actitud innovadora. En nuestra encuesta existe una alta presencia de agricultores con actitud innovadora, que no consideramos representa necesariamente al agricultor valenciano, pero si denota qué características se observan en los agricultores más innovadores. Es interesante constatar que la actitud innovadora se ve muy asociada al nivel de formación y que la presencia en zonas rurales, hoy por hoy, suponen un handicap. Son aspectos que las políticas educativas y de desarrollo rural deberían tener en cuenta.

Así pues, el productor innovador es aquel que muestra implicación en su actividad y lo demuestra dedicándole más de la mitad de su tiempo a la explotación. También favorece el comportamiento innovador el que toda o parte de la explotación se encuentre en régimen de arrendamiento. De igual manera favorece la innovación el contar con mano de obra asalariada.

Todos estos factores indican el intento por parte del sector de paliar uno de los problemas estructurales del sector como es la agricultura a tiempo parcial. A lo largo de las décadas la deriva de la agricultura hacia este modelo productivo permitió mantener la estructura minifundista y que el productor adoptase una postura diferente respecto a la explotación, asumiendo otras ocupaciones como su actividad principal.

El minifundismo también es un aspecto que va asociado a la agricultura parcial, y con ellos la desprofesionalización del sector. Asumir el régimen de arrendamiento para la explotación puede ser reflejo de cómo se intenta alcanzar un tamaño necesario para aprovechar las economías de escala. Lo que evidentemente precisa de un productor profesional enteramente dedicado a la actividad.

La agricultura a tiempo parcial también tuvo como consecuencia el auge de organizaciones cuyo objetivo era proveer los servicios como consecuencia la desprofesionalización del sector, ya que el productor veía posible llevar a cabo la actividad agraria compaginándola con la actividad en otros sectores.

Sobre este aspecto, el pertenecer a una organización es un aspecto que, en nuestra investigación, favorece la innovación, sobre todo en el caso de las cooperativas o SATs. Así, podemos constatar que la estructura organizacional valenciana nació y floreció con un objetivo, y hoy en día es un factor sumamente útil para mantener a la agricultura valenciana en la economía moderna. No obstante no hay que olvidar que la agricultura parcial funcionó en su momento dando salida a 
problemas como este, que son consecuencia de las carencias estructurales del sector, pero en la actualidad dejamos constancia de la necesidad de profesionalizar la agricultura valenciana.

Los resultados obtenidos en cuanto a formación hacen hincapié en la necesidad de contar con responsables empresariales profesionales. A mayor educación mejor predisposición muestra el productor a adoptar una actitud innovadora.

Según la orientación productiva vemos que las explotaciones que se dedican al cítrico o al cultivo de hortícolas tienden a situarse en el grupo de innovadoras. Puede reforzar esta impresión el hecho de que sean sectores muy orientados al mercado, con una vertiente exportadora notable. Pero por otra parte, las explotaciones que cuentan con una infraestructura de riego también son explotaciones con predisposición innovadora. Este hecho puede poner de relieve que la agricultura litoral se diferencia de la agricultura de interior, apareciendo la litoral más integrada en la dinámica económica actual. Ello sugiere la necesidad de adoptar políticas de desarrollo rural que revitalicen la diversificación económica en zonas de interior, teniendo en cuenta también el reto de la sostenibilidad.

Por último, en el grupo innovador están muy presentes explotaciones que pertenecen que obtienen un margen bruto anual superior a $50.000 €$ anuales. Esta tendencia muestra la inseguridad financiera que padece el sector e ilustra el hecho de que se asocie el adoptar innovaciones con la inversión de dinero. No queremos decir con esto que ser pequeña condene la explotación a ser débilmente innovadora, pues es un conjunto de características las que acaban influyendo en el comportamiento innovador.

Con todo ello, el contenido del presente trabajo evidencia el esfuerzo por parte del productor valenciano innovador de hacer frente a los problemas tradicionales que aquejan al sector. La Educación aparece como uno de los factores clave sobre el cual hay que seguir incidiendo para hacer viable el sector. 


\section{REFERENCIAS}

Alba, M. F., López-García Usach, T. García Álvarez-Coque, J.M. y Mas Verdú, F. [2010]. Conocimiento incorporado y vínculos sectoriales. El caso de una región con baja capacidad de absorción. International meeting on regional science. The future of cohesion policy. XVI reunión de estudios regionales. AECR. Badajoz, del 17 al 19 de noviembre de 2010. Recuperado, 1 de diciembre de 2018:https:/ / old.aecr.org/web/congresos/2010/htdocs/pdf/p172.pdf

Alba, M. F., [2012]. Conocimiento incorporado y vínculos intersectoriales. Aproximaciones mediante el análisis input-otput.Tesis doctoral. Valencia: Universitat Politècnica de València. Recuperado, 1 de diciembre de 2018: https://riunet.upves/bitstream/ handle/10251/15181/tesisUPV3754.pdf?sequence=1

Arnalte Alegre, E. [1989]. Estructura de las explotaciones agrarias y externalización del proceso productivo: implicaciones para el debate sobre el proteccionismo. Información Comercial Española, 666, 101-117

Boix, R. y Galleto, V. [2008). Marshallian Industrial districts in Spain. Scienze Regionali, 7 [3], 29-52.

Connor, J.M. y Schiek, W.A. [1997]. Food processing: an industrial owerhouse in transition. New York: John Wiley \& Sons [2aㅡ ed.].

Corbetta, P. [2003]. Metodología y técnicas de investigación social. Madrid: McGraw Hill.

López-Estornell, M. [2010]. Empresa Innovadora, Conocimiento y Distrito Industrial.Tesis Doctoral. Valencia: Universitat Politècnica de València.

López-García Usach, T. [2013]. Comportamiento innovador del sistema agroalimentario en la comunidad valenciana: cadena de valor, vínculos intersectoriales y territorio. Tesis doctoral. Valencia: Universitat Politècnica de València.

Instituto Nacional de Estadística.Censo Agrario [1999-2009]. Recuperado: http:/ / www.ine.es

Pérez-Gil, J. A., Chacón, S. y Moreno, R. [2000). Validez de constructo: el uso de análisis factorial exploratorio-confirmatorio para obtener evidencias de validez. Psicothema, 12, 2, 422-446. 